10

FINAL REPORT

PROJECT No. $1-173-80$

\title{
Development of Gene Transplant Technology for Crop Plants
}

J.S. Beckmann, Y. Salts, S. Izhar, R.W. Davis

$$
\begin{aligned}
& 630.72 \\
& \text { 2nd copy }
\end{aligned}
$$


14325

Plant genetics plait i mutation plant translocation

Petunia hyluida - Genetics Tomatoes - Genetics Maize - Genetics

$$
\frac{6,300,72}{B A R / B E C}
$$

and copy 


\section{A4325 To}

Standard BARD Cover Page for Scientific Reports

B ARD

January 1986

P.O.Box 6

Bet Dagan 50-250, Israel

BARD Project No. 1-173-80

Title of the Research Project: .

\section{Development of Gene Transplant Technology for Crop Plants}

Principal investigator: Jacques S. Beckmann

Cooperating investigators: Ronald W. Davis

Yehiam Salts*

Shamay Izhar"

Name and Address of Affiliated Institutions:

Principal Investigator: Dept of Plant Genetics and Breeding *, ARO, The Volcani Center, Bet Dagan 50-250, ISRAEL

Cooperating Institution: Dept of Biochemistry

Stanford University Medical Center

Stanford, Ca 94305, U.S.A.

Project's starting date: April 1981

Type of report: 1st Annual 2nd Annual Final $X$

Signatures:

Principal investigator

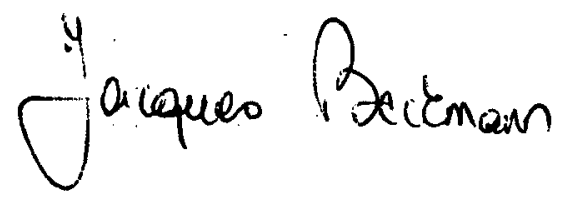

Institution's Authorizing Official

$$
\text { Yaval Eshlat }
$$

Dr. YUVAL. ESHDAT

Head, Div. for Research Projects and Budgets Agriculfural Research Organization

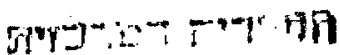

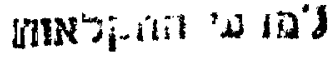

$$
\begin{aligned}
& \text { a } 2 \cdot 5-x \cdot 3
\end{aligned}
$$


b. TABLE OF CONTENTS

c. Abstract 2

d. Objectives 4

e. Body of the Report

1-1. A simple feeder-layer technique ....

I-2. Alternative selection systems: HAT, gpt, and canavanine 17

1-3. Mutant selection 26

I-4. Mutant characterization: $G-418^{R}$ and methotrexate ${ }^{R}$

1-5. Transient gene expression of the selectable marker 64

I-6. Physico-biological assays for penetration: $\quad 77$

II 1 RFLPs in tomatoes

II-2 RFLPs in maize

f. Description of cooperation: 143

g. Main Achievements 143

h. List of Publications 144

\section{c. ABSTRACT}

The purpose of this project was to apply recombinant DNA technology in plant systems. Two main issues were addressed. The first one concerns the development of possible routes to achieve gene transplant in plants. It involved:

(i) The development of suitable and efficient plating systems for single plant cells (Shneyour et al., 1983).

(ii) The isolation of specific plant cell mutants (Barg et al., 1984; Swartzberg et al., 1985; Shneyour et al., 1983). The selection schemes utilized were designed in such a way as to favor the selection of mutants arising preferably by a process of gene amplification (e.g. Schimke, 1984). A number of mutants were obtained, but, unfortunately, all our attempts to rescue the mutated loci from our petunia mutants in the form of regenerated whole plants failed.

(iii) Preliminary characterization of some of the methotrexate-resistant mutants demonstrated the unstable elevated nature of the dehydrofolate reductase activity. a property consistent with the expected gene amplification mechanism. Additional kinetic modifications were also observed (Barg et al., 1984 and $\mathrm{ms}$ in preparation). 
(iv) The development of several selection assays operable on plant cells. Both, plants and yeast, being sensitive to amitrole - an inhibitor of a key enzyme of the histidine biosynthetic pathway - raised the attractive possibility of exploiting the putative complementation in yeast, between yeast mutants and plant genes. Unfortunately, the lethal inhibitory mode of action of this compound on plant cells is too complex and prevented us to follow our initial plan. Other selection assays, for which heterologous vectors or genes were available, were tested for their suitability to our petunia cell system. Both the HAT and gpt selection proved to be unoperative with petunia. One system, the G-418 selection namely, seemed particularly attractive and was examined in details: It was discovered that this assay enables the utilisation of a selection for transient gene expression, a property which could be of utmost importance in recalcitrant cases. The possibility of exploiting the natural T-DNA encoded genes as selectable markers was also examined. The advantage here, is that it bypasses the need for smart vector design. It was shown that the natural Lysopine dehydrogenase could confer resistance to arginine analogues, presumably a consequence of this drug's conversion into a non-toric opine derivative.

(v) The testing of various routes to introduce macromolecules into plant cells. Attemps were made to challenge the synthesis of SV40 t-antigen in petunia protoplasts upon incubation of these cells with SV40 DNA under a variety of conditions. The fluorescent monitoring of the uptake by phagocytosis of CaP04 precipitates was no more successful. The capacity of Sendai virion envelopes to serve as microinjecting vehicles was also tested. Irreversible binding of these virions to the protoplast membranes could be demonstrated, provided Concanavalin A was added (Salts et al., 1985 ). This opens important possibilities regarding the potential of Sendai viruses as mediators in the transformation processes. Last but not least, microin jection through glass microcapillaries was also tested. Conditions were defined under which solutions could be microinjected into protoplasts without killing them. Such procedures already proved to be extremely useful in animal systems. Their potential in plant applications is also considerable. particularly if injections of large entities, such as organelles are considered. This is now the subject of a novel research project (P.I.: Y.Salts). 
The second part addresses the utilization of recombinant DNA for the genetic characterization of important crop species. Restriction Fragment Length Polymorphisms (RFLPs) were demonstrated to be an effective tool to this purpose. The first practical analyses were performed on tomatoes (ARO) and maize (Stanford). The applications of RFLPS in the area of cultivar and parentage identification, linkage studies, etc ..., as well as their extension to animal genetics, were considered also from a theoretical point of view. All these applications are now the subject of an additional project supported by BARD (1-397). In the present study, one particular aspect was also demonstrated, namely the usefulness of single corn probes in the genetic identification of the most commonly used breeding lines.

Barg R., N. Peleg, M. Perl and J.S. Beckmann (1984) Plant Mol. Biol. 3: 303-311.

Schimke R.T. (1984) Cell 37: 705-713.

Shneyour Y., A. Zelcer, S. Jzhar and J.S. Beckmann (1983) Plant Sci. Lett. 33: 292-302.

Swartzberg D., S. Izhar and J.S. Beckmann (1985) J. Plant. Physiol. 121: 29-35.

\section{d. OBJECTIVES}

Generally speaking, our aim was the development and introduction of the powerful molecular genetic engineering methodology for the manipulation of higher plants with the eventual goal, to render these techniques applicable to crop improvement.

Our initial proposal focussed thus on the development of routes for gene transplant technology, alternative to those involving Agrobacterium tumefaciens. The possibility of conferring novel or modified traits by single geen manipulation is expected to be of utmost importance in future breeding practice. This justified the initial selection of our objective. Our working rationale was, by analogy with knowledge gained from similar experiments in other biological systems, i.e., mammalian and yeast, that DNA uptake, in plants, is a normal process that occurs at very low frequencies, and hence, goes normally undetected. Since these events are the sine-qua-non for DNA transformation, special experimental conditions need to be designed to enable their recognition. The most sensitive assay, is a biological selection system, in which the transformed recipient cell, and it only, will survive and proliferate. This requires the utilization of 
specific donor genes conferring a selectable advantage to the recipient cells. The very low expected frequencies of such events, render obligatory the utilization of cloned genes of this kind. This requirement stands thus in sharp contrast to shotgunning experiments with total genomic DNA. Moreover, the absence of any molecular data pertaining to the actual structure or properties of plant controlling sequences such as promotors, splice sites, etc..., further limited the spectrum of available genes. Just as a reminder, no shuttle vector nor the likes had as yet been described, nor had any gene, but for the naturally occurring T-DNA derived ones, been shown to work in plant cells. Thus, in the absence of proper suitable selectable genes in plants, attention had to be directed i) to testing whether alien genes of this kind could be utilized, or else ii) to devise systems for the cloning of such plant genes, which would contain all the structural elements required for expression. Physical assays, monitoring for the uptake of alien material inside an isolated plant cell. though of limited usefulness, were also utilized, in order to optimize for penetration efficiency, or to devise alternative methods.

Since these operations require the handling of large cell numbers, as both protoplasts or cultivated cells, it was decided to concentrate on Petunia hybrida line 3704, for its apparent stability in tissue culture and general convenience, as judged by our laboratory's past experience. This is a diploid plant, from which suspension cell-lines have routinely been derived, from which protoplasts are readily obtained. It was used and proved to be extremely convenient a substrate, in a large somatic hybridization research program (e.g. Izhar et al., 1983).

Our first gal was thus the development of highly efficient plating assays, that would allow for the proliferation and colony formation. upon selection. of the very rare surviving cells. Likewise, a variety of potential candidate selection systems were scored and calibrated for their suitability to our plant material. Indeed, as more and more powerful selection systems were reported in the literature, some of which seemed to operate within a very wide host range, enabling the development of multiple-host shuttle vectors, it became reasonable to verify to what extent these conditions also applied to our plant cells. 
Specific selection assays were developed for the isolation of plant cell mutants, that were to serve as a source for the molecular cloning of the desired selectable gene-marker. Most available plant cell mutants were unfortunately useless for our purposes, for their lack of convenient suitable schemes that could lead to the molecular isolation of the marker-gene. The specific choice for the mutant selection was thus guided, mostly, by the availability of a scheme that would enable the cloning of the desired gene.

In the absence of cloned marker-genes and of suitable plant vectors, attempts were also directed at the improvement of insertion methods, using either physical or biological methods. Here again, our selection was guided by previous reported successes in heterolagous systems.

The following describes our research data in these directions. Most of it is presented in the form of reprints of published work, some of it as reports, some as yet unpublished data.

During the research period of this project. $\underline{\text { A. }}$ tumefaciens-mediated transformation enjoyed a tremendous boom. Thus, a reassessment of our goals had to be made. Indeed, in 1983, the generation of transgenic plants, in which a variety of foreign genes could be introduced and expressed was successfully achieved in a number of laboratories (e.g. Caplan et al, 1983). What seemed as the major bottleneck towards the implementation of molecular genetics to crop improvement appeared thus to be underway of being solved. The question had to be addressed as to where lie the new major problems in this area. We quickly came to realize that possessing the know-how for gene transfer is still a long way from its actual implementation in dayly practice. Major questions remained unanswered. Not the least being our lack of knowledge regarding what. specific genes to transfer, and more importantly, how to lay one's hands on these genes. This question became a significant part of our effort, and even developed into a novel independent research area, also supproted by BARD (1-397): Molecular genetics, namely, was proposed as an additional tool, via the use of Restriction Fragment Length Polymorphisms, for the genetic identification of crop plants (tomato and maize), with practical applications in the area of fingerprinting, parentage identification, all important in breeders' rights, and in the detailed 
genetic analyses of quantitative traits. the early stages of this research are described in part two of the Body of the report.

Caplan A., L. Herrera-Estrella, D. Inze, E. Van Haute, M. Van Montagu, J. Schell and P. Zambry shi (1983) Science 222: $815-821$

Izhar S.. M. Schlicter and D. Swartzberg (1983) Mol. Gen. Genet. 190: 468-474.

e. BODY OF THE REPORT

1-1. A simple feeder-layer technique for the plating of plant cells and protoplasts at low densities, and the isolation of $6-a z a-U$ and G-418 resistant mutants.

1-2. Alternative selection systems: HAT, $\mathrm{gpt}$, and canavanine

1-3. Mutant selection: Resistances to a minotriazole, and methotrexate

1-4. Mutant characterization: $G-418^{R}$ and methotrexate ${ }^{R}$

1-5. Conditions for transient gene expression of the selectable marker

1-6. Physico-biological assays for penetration: SV 40 antigen, Sendai virus, microinjection

II-1 RFLPs in tomatoes

II-2 RFLPs in maize

II-3 RFLPs as genetic markers (Madrid \& in hebrew) 


\section{1-2 Alternative selection systems: HAT, $8 \mathrm{pt}$, and canavanine.}

\section{1-2a BAT and get selections (R. Barg and J.S. Beckmann):}

All our attempts to apply to Petunia cells the Hypoxanthine-aminopterine-thymidine (HAT) selection system, or for that sake the gpt selection (Mulligan and Berg. 1981) failed: the presence of $10 \mathrm{uM}$ methotrexate (MTX) in the medium is tethal to these cells, even when hypoxanthine (at li! $U \mathrm{M}$ ) and thy midine (at $5 \mathrm{UM}$ ) are included. Higher thymidine concentrations were not tested since they are toxic to these cells. The lethality of this combination was unaffected by the order of addition of these components: preculturing the cells for 3 or $\theta$ days in the presence of hypoxanthine and thymidine, prior to the addition of the drug did not diminish lethality. None of a variety of compounds tested could attenuate the effects of MTX, when added to the HAT medium These include: non-essential amino acids (1\% final), as well as glycine or methionine (at 5 uM each), all added to the medium prior or together with the MTX. It should be pointed out, however that a recent study suggests that higher methionine concentrations ( $20 \mathrm{uM}$ ) diminish the toxicity of MTX (Marion-Poll et al., 1984). Addition of $5 \%$ fetal calf serum to the selection medium also failed to reverse the MTX effect

In attempts to elucidate the failure of petunia cells to grow in HAT medium, the capacity to take up thymidine, or other components of these selection systems was measured Cells were cultured for $24 \mathrm{hrs}$ in the presence of $3 \mathrm{H}$-thymidine. the latter was found to be incorporated into ice-cold TCA precipitable material (presumably DNA). ${ }^{14} \mathrm{C}$-labeled hypozanthine was also found to be incorporated into ice-cold TCA precipitable material. Similar experiments were also performed with ${ }^{14} \mathrm{C}$-labeled ranthine, but in this case no radioactivity could be recovered in the precipitate. It should be mentionned that this last experiment was problematic, since because of the solubility properties of xanthine, a high pH solution was fed to the cells, which in itself might have inhibited incorporation.

Conditions which enable to perform HAT selection in animal systems could not be reproduced with petunia cells. Nor could any of a variety of compounds tested reverse the loxicity of 
MTX to these cells. The establishment of such a selection system is a prerequisite for bath straightforward HAT selection as well as for the application of the more versatile gpt selection (Mulligan and Berg, 1981). Hence, we were forced to conclude that at present none of these selections could be applied in a simple way to petunia cells and presumably, to other plant cells as well. It should be pointed out, however, that with respect to the gpt selection assay. the effect of mycophenolic acid alone was not tested. This leaves thus this possibility for a gpt selective assay still an open issue

Marion-Poll A., C. Missonier, M.-A. Grandbastien and M. Caboche (1984) P1. Sci. Lett. 36 : 169 170 Mulligan R.C. and P. Berg (1981). Proc. Nati. Acad. Sci. USA 78: 2072-2075. 


\section{f. Description of cooperation}

Information and plans were exchanged between the two laboratories. J.S. Beckmann visited Stanford twice during this period. R. W. Davis and J.S. Beckmann also met once in Europe, during a conference. This was particularly important for the research on RFLPs. Indeed, these projects had started independently in both laboratories. The Stanford Lab had been involved in the initial human RFLP work, and brought this contribution to the attention of the ARO's team.

\section{g. Main Achievements}

Basic steps, required for the implementation of the powerful genetic engineering methodology to agriculturally important crop species, were addressed during this project. These concentrated mostly on the various ways towards the generation of transgenic plants. Some of the results obtained, such as the possibility of "induced gene amplification" in plants - in this case of a housekecping gene - and the development of microinjection have opened new research avenues. with many important ramifications. In addition given the significant development in $\mathbf{A}$. tumefaciens mediated transformations, a general reassessment of priorities was made. This led to the introduction of a novel tool in the area of plant breeding. "Restriction Fragment Length Polymorphism" namely. The first practical and theoretical steps of the novel RFLP methodology were examined. These are now the subject of an additional project supported by BARD. 


\section{h. List of publications}

Barg Rivka, Jacques S. Beckmann and Meir Perl (1984) An NADPH-Dependent Irreversible Methotrexate-Binding in vitro to a Protein Fraction from a Methotrexate-Resistant Petunia Cell-Line. Published in the Abstracts of the 4th Congress of the Federation of European Societies of Plant Physiology. Strasbourg 1984, p211.

Barg Rivka, Naomi Peleg, Meir Perl and Jacques S. Beckmann (1984) Isolation of methotrexateresistant cell lines in Petunia hybrida upon stepwise selection procedure. Plant Mol Biol 3 303-311.

Bar-Nun Shoshana, Yonah Shneyour and Jacques S. Beckmann (1983) G-418, an elongation inhibitor of 805 ribosomes. Biochim. Biophys. Acta 714: 123-127.

Rom Moshe (1982) Development of Restriction Fragment Length Polymorphism Techniques in Plants as a Tool for Strain Identification and Breeding. M.Sc. Thesis (in Hebrew with English summary). The Hebrew University. Jerusalem, Israel.

Rom Moshe and Jacques S. Beckmann (1985) DNA polymorphisms: A new tool for genetic identification and mapping (in Hebrew). Mada (Science) 29: 123-128.

Salts Yehiam, Jacques S. Beckmann, Avraham Loyter, and Uri Lavi (1985) Interaction of Sendai Virus with Plant Protoplasts. Plant Sci. 41: 141-149.

Shneyour Yonah. Aaron Zelcer, Shamay lzhar and Jacques S. Beckmann (1983) A simple feederlayer technique for the plating of plant cells and protoplasts at low densities. Plant Sci. Lett. 33: 292-302.

Swartzberg Dvora, Shamay Izhar and Jacques S. Beckmann (1985) Tobacco Callus Line Tulerant to Amitrole: Selection. Regeneration of Plants and Genetic Analysis. J. Plant. Physiol. 121 : 29-35. 